\title{
Compressive, shear, and fracture behavior of CNT reinforced Al matrix composites manufactured by severe plastic deformation
}

\author{
Hassan Zare ${ }^{\text {a }}$, Mohammad Jahedi b,*, Mohammad Reza Toroghinejad a , Mahmoud Meratian ${ }^{\text {a }}$ \\ Marko Knezevic ${ }^{\mathrm{b}}$ \\ a Department of Materials Engineering, Isfahan University of Technology, Isfahan 84156-83111, \\ Iran. \\ ${ }^{\mathrm{b}}$ Department of Mechanical Engineering, University of New Hampshire, Durham, NH 03824, \\ USA.
}

\begin{abstract}
In this work, carbon nanotubes (CNTs) reinforced aluminum (Al) matrix composites are synthesized using $\mathrm{Bc}$ equal-channel angular extrusion (ECAP) route and their mechanical behavior is examined under compression and shear deformation. The results show that at room temperature, eight ECAP passes are necessary to achieve the density of the composite where the effect of CNTs in enhancing the mechanical properties become significant. Samples of pure Al are also processed under the same ECAP conditions, and their properties are further examined to facilitate the comparison. The well-densified composites with only 2 vol. \% of CNTs exhibit an approximately $30 \%$ increase in yield strength compared to the pure Al samples. Microstructure data in terms of porosity volume fraction, crystallite size, and dislocation density, along with the residual lattice strain measurements, are used to explain the observed improvements in strength. As measured by X-ray diffraction (XRD), higher levels of dislocation density, smaller crystallite sizes, and larger residual lattice strains are present in Al-CNT than in pure Al samples. Finally, fractographic analysis using scanning electron microscopy is performed revealing that the fracture surfaces of the composite exhibit a more brittle behavior than pure Al samples.
\end{abstract}

Keywords: Metal matrix composites (MMCs); Severe plastic deformation (SPD); Shear punch test (SPT); X-ray diffraction (XRD); Fractography

\footnotetext{
${ }^{*}$ Corresponding author at: Department of Mechanical Engineering, University of New Hampshire, 33 Academic Way, Kingsbury Hall, W322, Durham, NH 03824, USA.

E-mail address: mohammad.jahedi@unh.edu (M. Jahedi).
} 


\section{Introduction}

During the past four decades, the emergence of a wealth of literature concerned with the processing, microstructure, and properties of discontinuously reinforced metal matrix composites (MMCs). Reinforcements such as silicon carbides ( $\mathrm{SiC})$ [1-3], alumina $\left(\mathrm{Al}_{2} \mathrm{O}_{3}\right)$ [4], and boron carbide $\left(\mathrm{B}_{4} \mathrm{C}\right)[5]$ are regularly used in combination with a matrix needing an enhanced property or a set of properties. A less commonly used reinforcement is a carbon nanotube (CNT) [6]. CNT as one of the carbon allotropy has unique properties arising from their symmetric crystal structure. Mechanical properties of CNTs are far beyond the mechanical properties of any matrix material making them an excellent reinforcing material within an appropriate matrix material. Improvements of matrix materials by CNTs in terms of strength $[7,8]$ as well as functional properties [9] have been demonstrated. These materials have yet to be commercialized. In addition to being able to overcome the factors associated with the cost of the CNT reinforced composites, there is a lack of confidence in these materials due to the presence of porosities, low interfacial bond energy between matrix and reinforcements, and most importantly, poor distribution of reinforcements. Therefore, there is a critical need to overcome these shortcomings by studying the processing methodologies and characterizing properties of the outcome material.

Conventional deformation processes such as rolling and extrusion have been utilized extensively in the consolidation of the powders containing reinforcing particles [10, 11]. It was found that at room temperature, improving density of the consolidated materials and the material homogeneity requires very high strains $(>4)$. The processes of severe plastic deformation (SPD) have attracted a lot of attention due to their ability to impart large plastic strains. In general, the larger strain levels result in the better homogeneity of particle distribution in the material. However, more energy and time are needed. While SPD processes such as equal channel angular pressing (ECAP) [12, 13], accumulative roll bonding (ARB) [14-17], and high-pressure torsion (HPT) [18] have been found to improve the consolidation process and distribution of the particles, several other innovative methods such as torsion extrusion (TE) [19], ECAP with back pressure [20], ECAP incorporating with torsion (T-ECAP) [21], and high pressure double torsion (HPDT) [3] have been even more effective. In particular, HPDT was found to enhance distribution of $\mathrm{SiC}$ in a $\mathrm{Cu}$ matrix [3] as well as to improve crystallographic texture homogeneity [22]. Among these bulk SPD processes ensuring consolidation and particle dispersion, ECAP is 
the most attractive due to its simplicity and its ability to impair the most homogeneous strain distribution of all the alternatives.

The ECAP process has two channels with the same cross-sectional area. Samples are usually circular or square according to the dimensions of the channels. After applying the appropriate lubricants, the sample is placed in the inlet channel and extruded through the outlet channel using a punch under pressure. The underlining deformation mode in the ECAP process is a simple shear.

In this paper, Al-CNT composites are fabricated using a multi-pass ECAP processing method on 2, 4 and 8 passes. The content of CNTs was relatively low at only 2 vol.\%, which is added into the pure Al matrix. To disperse the CNTs in the matrix before the ECAP consolidation, a combination of attrition milling and ultrasonic waves was utilized. The created materials were then characterized in terms of microstructure and mechanical properties. First, density measurements were performed using the Archimedes method to evaluate the level of densifications. Next, the crystallite size, dislocation density, and residual lattice strains were measured using X-ray diffraction (XRD). Mechanical properties are tested in simple compression and shear punch test (SPT). Finally, fractographic analysis was performed using scanning electron microscopy (SEM). Using the fracture surface analysis [23, 24], the cooperative role of porosities and CNTs in the failure process was determined and ductile vs. brittle character of fractured surfaces [25-27] was characterized.

To facilitate the comparison of mechanical properties, pure Al samples were also processed in the same way. To process the material to the desired level of densification, 8 ECAP passes were necessary. The compression and shear strength measurements revealed the significant role of CNTs on enhancing the mechanical properties of the Al matrix. The strength of the Al-CNT composite was higher than the pure Al by approximately $30 \%$. The improvements were explained by a higher content of stored dislocations, smaller grain size, and a higher residual stress in the Al-CNT composite than in pure Al. CNTs act as obstacles to dislocation motion as well as hinder any grain growth during dynamic processes of recovery and recrystallization. Thus, CNTs help facilitate a finer grain structures in the composite than the pure material. The fracture surface analysis after SPTs revealed more ductile fracture features in the pure Al than in the Al-CNT samples, which behaved largely in a brittle manner. Interestingly, 
the CNTs were found to create a network connecting the particles and grain boundaries, which is an important factor enhancing mechanical properties of the composites.

\section{Materials and experimental procedures}

This section will begin by describing the starting materials and the fabrication procedures using ECAP for making the Al- 2 vol. \% CNT composite samples. Subsequently, it describes the mechanical testing procedures including compression and shear to quantify the material properties as well as the characterization techniques used including X-ray diffraction (XRD) and scanning electron microscopy used to explain the observed material behavior. Finally, the composite material samples are critically compared with the Al samples in the same condition.

\subsection{Materials}

Commercially pure aluminum powders with an average particle size of $30 \mu \mathrm{m}$ and multiwalled carbon nanotubes (MWCNTs) with a diameter of 10 to $30 \mathrm{~nm}$ and a length of 5 to $15 \mu \mathrm{m}$ are used as the starting materials. CNTs are produced with catalytic chemical vapor deposition (CCVD) method, which revealed $95 \%$ purity and density of $1 \mathrm{~g} . \mathrm{cm}^{-3}$. An acidic treatment was performed to remove catalytic impurities from the CNT powders, which are mainly nickel and cobalt. The procedure is to treat the CNTs in a solution of nitric acid of $68 \%$ purity for 12 hours. This process followed by washing the CNTs with distilled water several times to reach pH equals to 7. The powder's preparation procedure requires ultrasonic wave treatment during the entire attrition milling process. In the attrition milling, the ball to powder ratio (BPR) is approximately 15 and the milling speed was $500 \mathrm{rpm}$. The milling and ultra-sonication are continued until the mixture reaches a semi-solid paste. Finally, the mixture was heated at a low temperature $\left(50^{\circ} \mathrm{C}\right)$ to evaporate the remaining acetone from it. This procedure was found to disperse the CNTs well within $\mathrm{Al}$ before the consolidation using ECAP.

\subsection{ECAP processing}

The prepared mixture was consolidated using the equal channel angular pressing (ECAP) process of severe plastic deformation (SPD) at room temperature. The ECAP die had $20 \mathrm{~mm}$ channel diameter with the geometrical angles of $\varphi=120^{\circ}$ and $\psi=20^{\circ}$, where $\varphi$ is channel angle and $\psi$ is outer corner angle. The route $\mathrm{B}_{\mathrm{C}}$ was utilized, as it is known to impart better strain 
homogeneity than other ECAP routes and is more effective on the grain refinement [28, 29]. The ram speed was $0.8 \mathrm{~mm} \cdot \mathrm{sec}^{-1}$. The effective strain after each pass is estimated to be 0.67 using $\bar{\varepsilon}=\frac{2}{\sqrt{3}} \cot \left(\frac{\varphi}{2}\right)[30]$ with the die geometry used.

\subsection{Density measurement}

Density was measured by the Archimedes method, based on the ASTM C20.00. The samples were kept suspended in the distilled water at $100^{\circ} \mathrm{C}$ for 2 hours. To obtain the density of each sample, three mass values were required: mass in the dry state $\left(m_{d}\right)$; mass sample suspended in water $\left(m_{S u}\right)$; and the saturated mass $\left(m_{S a}\right)$. The density was calculated using, $\rho=\frac{m_{d}}{m_{S a}-m_{S u}}$.

\subsection{X-ray diffraction (XRD) analysis}

To measure the crystallite size, stored dislocation density, and residual micro-strain in the $\mathrm{Al}$ and Al-CNT samples, the X-ray diffraction (XRD) was utilized. The measurements were performed on the samples processed to 2, 4, and 8 passes of ECAP. In all tests, $\mathrm{Cu}$ Ka radiation, with a wavelength of $0.1542 \mathrm{~nm}$, was used. In general, two methods are typically used to interpret the XRD patterns: the Williamson-Hall method [31, 32] and the Rietveld method [33, 34]. The advantage of the latter method is the consideration of anisotropy, which is important for a material undergoing severe plastic deformation [35]. Here, the Rietveld method was utilized in calculating the microstructural parameters of crystallite size and dislocation density as well as the residual micro-strains for all the processed samples [36]. The analysis has been performed using the sophisticated software called Materials Analysis Using Diffraction (MAUD). In this software, a parameter called GOF (goodness of fit) was implemented to ensure the best fit for the measured XRD patterns in the laboratory. Acceptable accuracy was obtained when the GOF value was between 1 and 2 [37] and all the results presented in this work were within the bounds.

\subsection{Mechanical properties}

Since the dimensions of the created materials were relatively small $(14 \mathrm{~mm}$ and $20 \mathrm{~mm}$ in diameter and length, respectively), making simple tensile test specimens was not possible. Therefore, investigating the strength of the samples was performed based on the simple compression and shear tests. 


\subsubsection{Simple compression}

The cylindrical samples were machined out from the ECAP specimens with a height to diameter ratio of 1.5, with a diameter of $10 \mathrm{~mm}$ [21]. The yield stress determined in compression is believed to be an accurate measure of yield stress because of the face-centered cubic (FCC) structure. Typically materials do not exhibit appreciable tension/compression asymmetry [38, 39].

The simple compression experiments were performed on a 100 tonf compression hydraulic-testing machine. The machine was equipped with a compression fixture consisting of two cylindrical compression dies aligned vertically along the axis of the load cell. The compression dies are made of a AISI D3 oil hardened tool steel with smooth flat surfaces on one side produced by surface grinding and polishing. Prior to the experiments, the compliance of the machine was documented by compressing the dies without a specimen in order to record the load and displacement of the actuator. The compliance of the system was later used to determine the actual displacement of the specimen in compression. During the experiments, the load and displacement of the actuator were recorded. The tests were also performed at an initial strain rate of $0.01 \mathrm{sec}^{-1}$ at room temperature. The lubricant, Teflon tape with silicon spray [21], was primarily used to minimize the effects of the contact friction between faces of the specimen and the upper and lower compression dies. The raw data were collected in the form of load and displacement, and corrected for machine compliance [40] before computing the stress strain curves. The samples were compressed to failure. Five samples were deformed per category to ensure reputability and accuracy of the measured data.

\subsubsection{Shear punch test (SPT)}

A shear punch test (SPT) is a mechanical test in which a thin strip of specimen is clamped between a set of dies, which are blanked using a flat cylindrical punch at a low constant speed. The specimen deformation occurs in the small annular zone between the punch and the die. The shear yield strength (SYS), ultimate shear strength (USS), and shear elongation percentage $\left(\begin{array}{ll}\% & \varepsilon_{u}\end{array}\right)$ values can be calculated from the SPT data. A linear correlation between tensile and SPT shear strengths has been reported for some materials [41, 42]. In particular, Guduru et al. [4, 42] founded a linear correlation between the shear and tensile data for yield for 
the $\mathrm{Al}$ alloy. The relationship suggested is $\sigma_{0.2}=\alpha \tau_{1.00}$, where $\alpha=1.77$ and $\tau_{1.00}$ is the $1.00 \%$ SPT normalized displacement offset yield point.

Shear punch test (SPT) according to the following standards: BS 5669, BS 2782, and ASTM D-732, [42, 43] can be conveniently used to evaluate strength properties of materials whose dimensions are insufficient to make uniaxial tensile specimens. The shear punch apparatus consisted of an upper and lower housing. The upper housing contained a punch, and the lower one held a die. The material selected for both the punch and die was a AISI D3 oil hardened tool steel $(65 \pm 3 \mathrm{HRC})$. The central axes of the punch and die were exactly in the upper and lower housing; they were adjusted by the cross table installed at the bottom of the instrument. The specimen was placed between the die and a washer. A flat tip punch was forced through the specimen, and the act of punching against the die that sheared a circular disk from the specimen, completed the test.

In our SPT testing setup (Fig. 1), the sample thickness, $h$, varies between 100-1000 $\mu \mathrm{m}$. The recent study revealed that the optimum thickness range is recommended to be $400-600 \mu \mathrm{m}$ $[4,41,43]$. Here, the specimens of $500 \pm 20 \mu \mathrm{m}$ thickness were used. The specimens were cut into disks of $10 \mathrm{~mm}$ in diameter using a wafering saw. The top and bottom surfaces of the cylinders were then rough grinded with diamond lapping films and fully polished with $1 \mu \mathrm{m}$ diamond paste. Minimum three shear punch tests were carried out on each specimen at the room temperature holding the constant cross-head speed of $0.1 \mathrm{~mm} \cdot \mathrm{min}^{-1}$.

Shear stress is obtained using $\tau=\frac{P}{\pi \times d_{\text {avg }} \times h}$ where $d_{\text {avg }}=\left(d_{\text {punch }}+d_{\text {die }}\right) / 2, P$ is applied load, and $h$ is specimen thickness. The idealized (pure) shear zone is the red region between the die and punch that forms upon imposing a punch displacement $\delta$ [4, 41-43]. In our setup $d_{\text {punch }}=5.42 \mathrm{~mm}$ and $d_{\text {die }}=5.38 \mathrm{~mm}$.

The setup has certain compliances, which was extracted, and the elastic displacement $\left(\delta_{c}\right)$ was subtracted from the punch displacement $(\delta)$ to obtain the real punch displacement $\left(\delta_{r}\right)$ imposed over the tested sample. The resultant punch displacement $\left(\delta_{r}\right)$ divided by the specimen thickness $h$ yields the normalized displacement $\delta_{n}=\delta_{r} / h$, which will be used in plotting the SPT testing results [42]. 


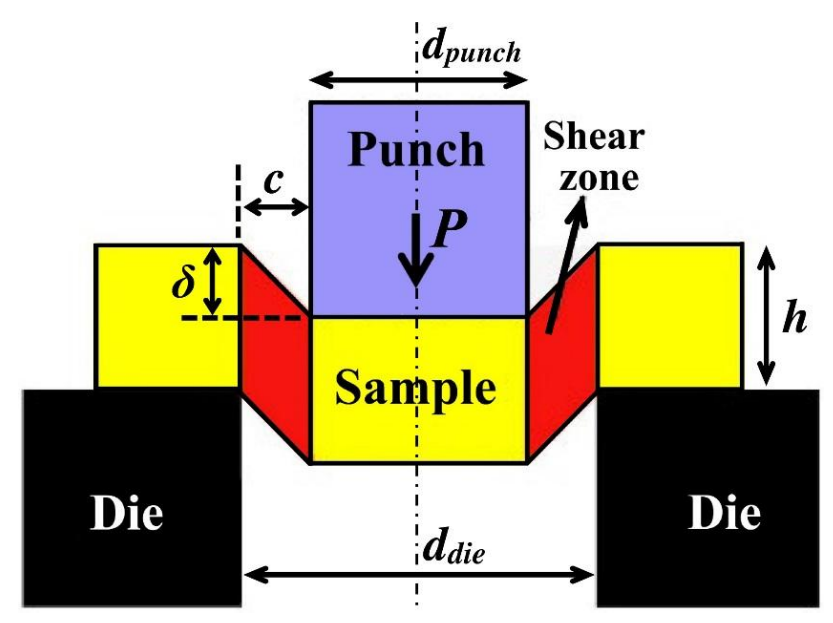

Figure 1 Schematic showing the shear punch testing setup.

\subsection{Fracture surface analysis}

The fractured surface investigation of the samples after SPT was performed to determine the role of porosities and CNTs within the failure mechanisms of $\mathrm{Al}$ and in the composite. The samples processed to different number of ECAP passes were examined after failing in shear. To facilitate this, scanning electron microscope imaging in (SEM) PHILIPS XL30 model was performed.

\section{Results and discussion}

\subsection{Density measurements}

The density measurement results are summarized in Table 1 . The standard deviation is also shown, which was determined based on measurements of multiple samples per category. It can be seen that the density of pure Al consolidated samples is always higher than Al-CNT samples. This is due to the incoherency of CNT particles with the Al matrix leading to the pores around the CNT particles. The presence of CNTs leads to less breaking of the powder surface ridges and less penetrating into the pores spaces [44]. These factors reduce the ability of the material to flow plastically, achieving a fully dense material structure [45]. 
Table 1 Relative density (in percentage) of ECAPed samples after different number of passes.

\begin{tabular}{|c|c|c|c|}
\hline Samples & Pass 2 & Pass 4 & Pass 8 \\
\hline Al & $92 \pm 0.5$ & $96 \pm 0.4$ & $98.5 \pm 0.35$ \\
\hline Al-CNT composite & $91 \pm 0.7$ & $94.5 \pm 0.65$ & $97 \pm 0.4$ \\
\hline
\end{tabular}

\subsection{XRD measurements}

Rietveld method simulates the experimental XRD patterns by computing functions that take into consideration the structural parameters as well as the device parameters including: (1) peak shape which is influenced by the characteristics of the beam; (2) the experimental arrangement and the sample size and shape; (3) peaks width, which broadens; (3) and preferred orientations, which result in a greater volume fraction of certain crystal orientations. All the variables are optimized and refined by repeating the method of the least squares and by minimizing the remaining parameters.

Several factors contribute to the observed peak broadening $\quad\left(\mathrm{B}_{\mathrm{obs}}\right), \quad B_{\text {obs }}=B_{\text {instr }}+$ $B_{\text {sample }}=B_{\text {instr }}+B_{\text {size }}+B_{\text {strain }}[46,47]$. The instrumental broadening can be determined experimentally with a diffraction standard or calculated with the fundamental parameters approach. Scherrer in 1918 suggested an equation for measuring the broadening due to size which is $B_{\text {size }}=K \lambda / D \cos \theta$ where $D$ is volume average of crystal thickness in a direction normal to reflecting planes (which simply called crystallite size), $\theta$ is diffraction angle of the reflection, $\lambda$ is the wavelength, and $K$ is constant of proportionality. Lattice strain (micro-strain) which arises from the displacement of the unit cells from their normal positions is usually quantified as $\varepsilon_{0}=\Delta d / d$, with $d$ the idealized 'd spacing' and $\Delta d$ the most extreme deviation from $d$. The peak broadening due to strain can be simplified by $B_{\text {strain }}=4 \varepsilon_{0} \tan \theta[48,49]$. In all other sophisticated models, the peak broadening due to size and strain are assumed to vary as $B_{\text {size }} \propto 1 / \cos \theta$ and $B_{\text {strain }} \propto \tan \theta$, respectively.

Particles of powder are usually comprised of multiple grains having different sizes and crystal orientations. During the consolidation process, the particles: (1) bond together, which 
leads to the formation of new interfaces in the microstructure and (2) fragment, which leads to the formation of new grains that have different crystal orientations and grain boundaries. These small new grains inside particles are referred to as crystallites. It should be noted that the new grain boundaries inside the initial particles have different energy values than the bonding interfaces. It is thus possible to separate the crystallite size by the XRD measurement.

By fitting the XRD patterns, the MAUD software estimates the crystallite size and residual micro strains. Figure 2a shows the changes in crystallite size of Al and Al-CNT samples after a number of different passes of the ECAP process. As expected, the crystallite sizes decrease with the increasing number of passes in both $\mathrm{Al}$ and Al-CNT samples. However, the crystallite size is reducing more rapidly in the composite than in the pure Al samples. CNTs in the matrix influence the glide of mobile dislocations and also hinder the dynamic recovery processes known to operate in the high-stacking fault Al. The overall effect is a higher content of stored dislocations in the composite than in pure Al samples. The higher content of stored dislocations lead to more grain fragmentations during the ECAP processing of the composite than the pure metal. 

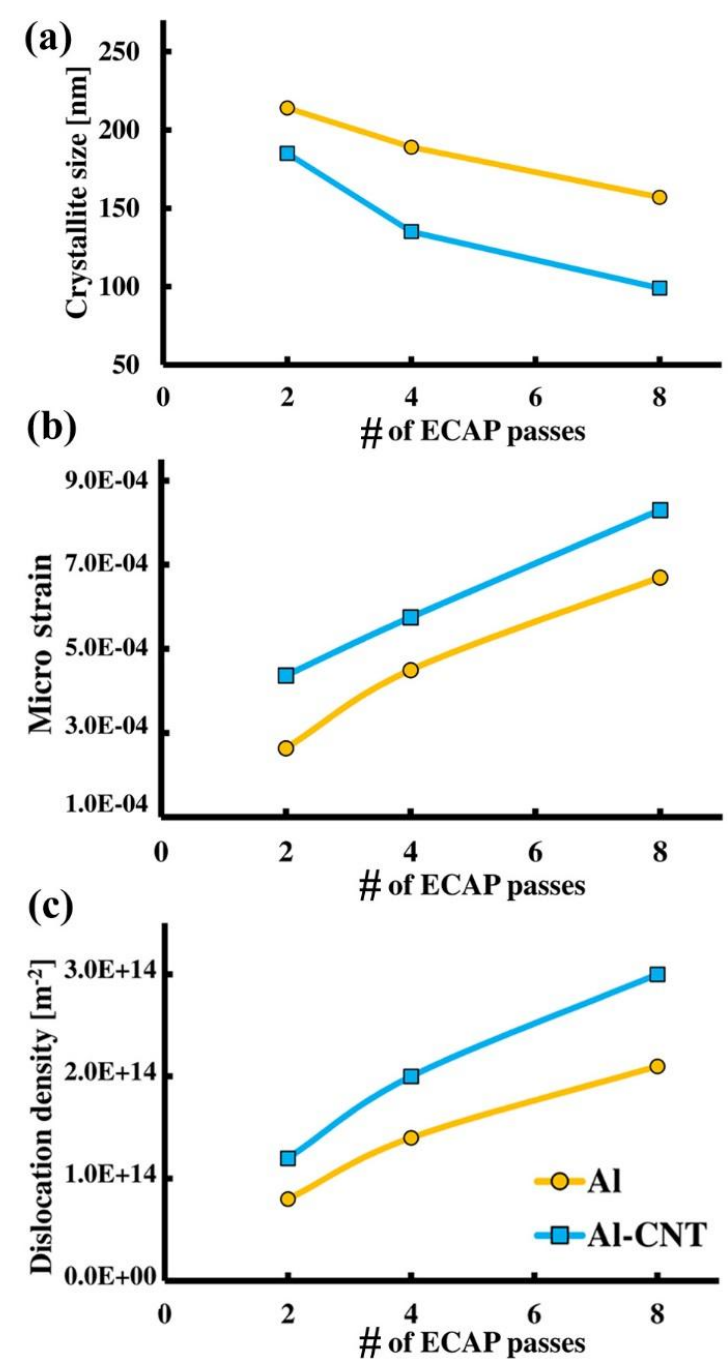

Figure 2 (a) Crystallite size, (b) residual micro strain, and (c) evolution of stored dislocation density in pure $\mathrm{Al}$ and $\mathrm{Al}-\mathrm{CNT}$ processed to different number of ECAP passes.

Many researches $[50,51]$ reported that microstructural changes during the process of ECAP are based on the theory of grain subdivision where the grain separation is happening by stored dislocations created boundaries. As a result of plastic deformation, the forest dislocation density is increasing and the structure of these randomly distribution dislocations is gradually becoming organized into cell walls and micro shear bands $[52,53]$. As a result, the so-called geometrically necessary boundaries (GNBs) are created [54]. These GNBs are becoming high angle grain boundaries with the continuation of plastic deformation, and thus, facilitate the grain subdivision mechanism. In the ECAP process, GBNs are initially aligned with the axis of 
deformation and then the distance between them decreases with an increase in misorientation $[54,55]$.

To calculate the average density of dislocations $(\rho)$, the following relations were used;

$\rho=\sqrt{\rho_{D} \times \rho_{S}}$ where $\rho_{D}=3 / D^{2}$ and $\rho_{S}=6 \pi \times \varepsilon_{L}^{2} / b^{2} \cdot \rho$ is the dislocation density while $\rho_{D}$ and $\rho_{S}$ dislocation density according to the crystallite size and strain, respectively. $D$ is average crystallite size, $\varepsilon_{L}$ is average micro strain, and $b$ is Burgers vector.

The micro strain changes are shown in Fig. 2b for both the Al and Al-CNT samples. Evidently the micro strains increase with the number of ECAP passes.

Figure 2c shows evolution of dislocation density in the Al and Al-CNT-reinforced samples after ECAP processing. High dislocation density causes creation of sub boundaries and refinement in grain structure. In addition, CNTs reduce the mean free path of mobile dislocations. As a result, a higher dislocation density content is observed in the Al-CNT samples than in the Al samples.

\subsection{Compression test}

Figure 4 shows results of the compression test of the consolidated powder samples. As this figure shows, both $\mathrm{Al}$ and composite samples processed to 2 and 4 ECAP passes fail after a short work hardening, which is manifested by the drop in stress value. The amount of work hardening of the samples made using 4 passes was improved over those made using 2 passes. The compressive flow curve of the 8th passes samples is substantially longer, exhibiting strainhardening characteristics. The fact that the 8 passes curve exhibit the work hardening behavior is the signature of the dense material where there is a competition between dislocation generation and recovery processes. This shows the effectiveness of the ECAP process to make the composite materials of superior mechanical properties. However multiple ECAP passes are necessary to make sufficiently dense materials. At least 8 ECAP passes was found to be necessary in making the sufficiently dense samples.

For reference, the flow stress of the pure bulk Al manufactured using 8 ECAP passes is shown. The data was taken from [56]. Evidently, the bulk material shows more work hardening 
and a higher ultimate stress than the consolidated pure $\mathrm{Al}$ from powder. This can be attributed to the fact that the consolidated samples contain porosities that weaken the matrix. Most of the plastic deformation imposed on the sample collapse the porosities, and as a result, a lesser amount of plastic strain goes into the powder samples than in the bulk material samples for a given number of passes. Therefore, the bulk material is expected to have a finer grain structure resulting in a higher flow stress.

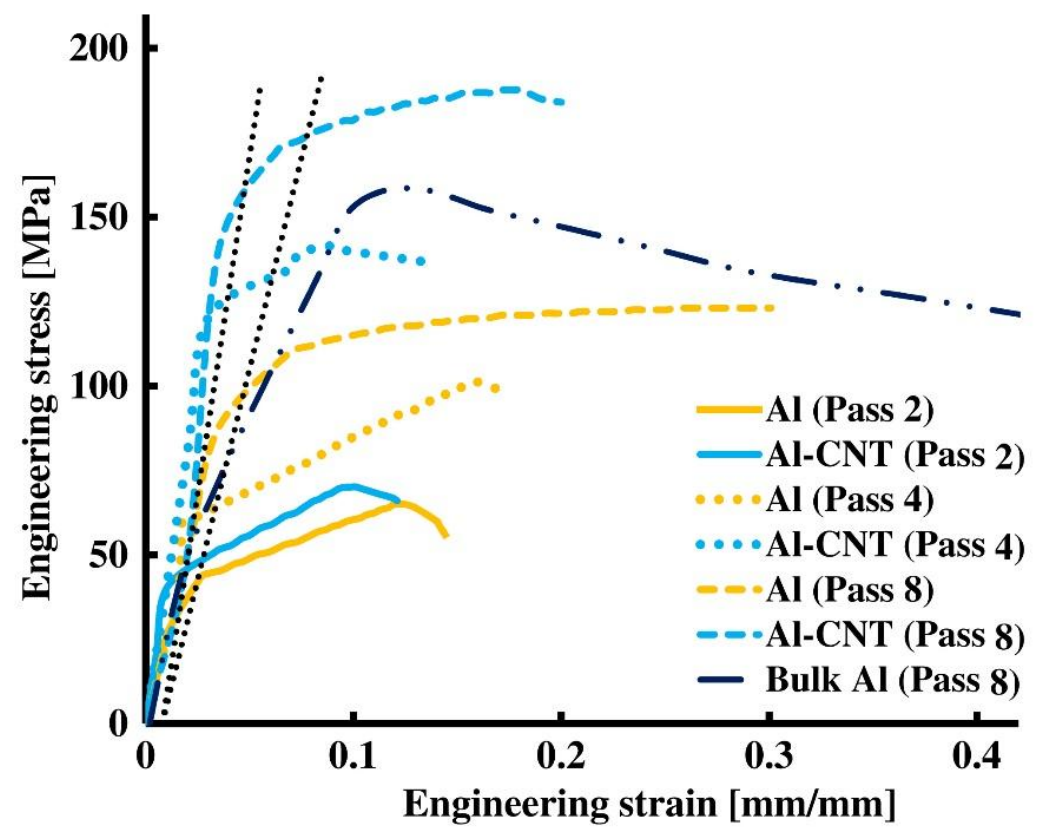

Figure 4 Compressive engineering stress- strain curves for $\mathrm{Al}$ and $\mathrm{Al}-\mathrm{CNT}$ composite samples tested after a different number of ECAP passes as indicated in the legend. The dotted lines show the $0.2 \%$ offset.

In Fig. 4, an increase in the strength and elastic modulus of the composite samples can be observed and compared to the samples of Al. This is due to the CNT reinforcement content and the underlining strengthening mechanisms. Introducing the CNT interfaces between the CNTs and the matrix produces more dislocations in the composite sample, as CNTs act as obstacles to the motion of mobile dislocations. The created pile-ups lead to an increase in the work hardening, which results in the overall increase in the strength of the composites. Moreover, the CNT-reinforced composites have finer grain size that pure Al processed using the same number of ECAP passes [57, 58]. The hardening mechanisms overcome the effect of the larger content of porosities in the CNT-reinforced composite than in the pure Al samples. Finally, it should be 
noted that adhesion between the particle and the matrix results in a strengthening via the load transfer mechanism, which is due to the volume fraction and the aspect ratio of particles in a MMC. This eventually leads to a better transfer of stress from the matrix to the CNTs [59, 60]. The failed samples after compression tests are shown in Fig. 5. As can be seen, the samples cracked and broke in a brittle manner. The Al samples have less destruction than the composite samples, which can be associated with the higher density of the composite sample than $\mathrm{Al}$ one. The 8th pass Al sample retained integrity and some aspects of a ductile flow. This figure demonstrates the brittleness of the composite samples.
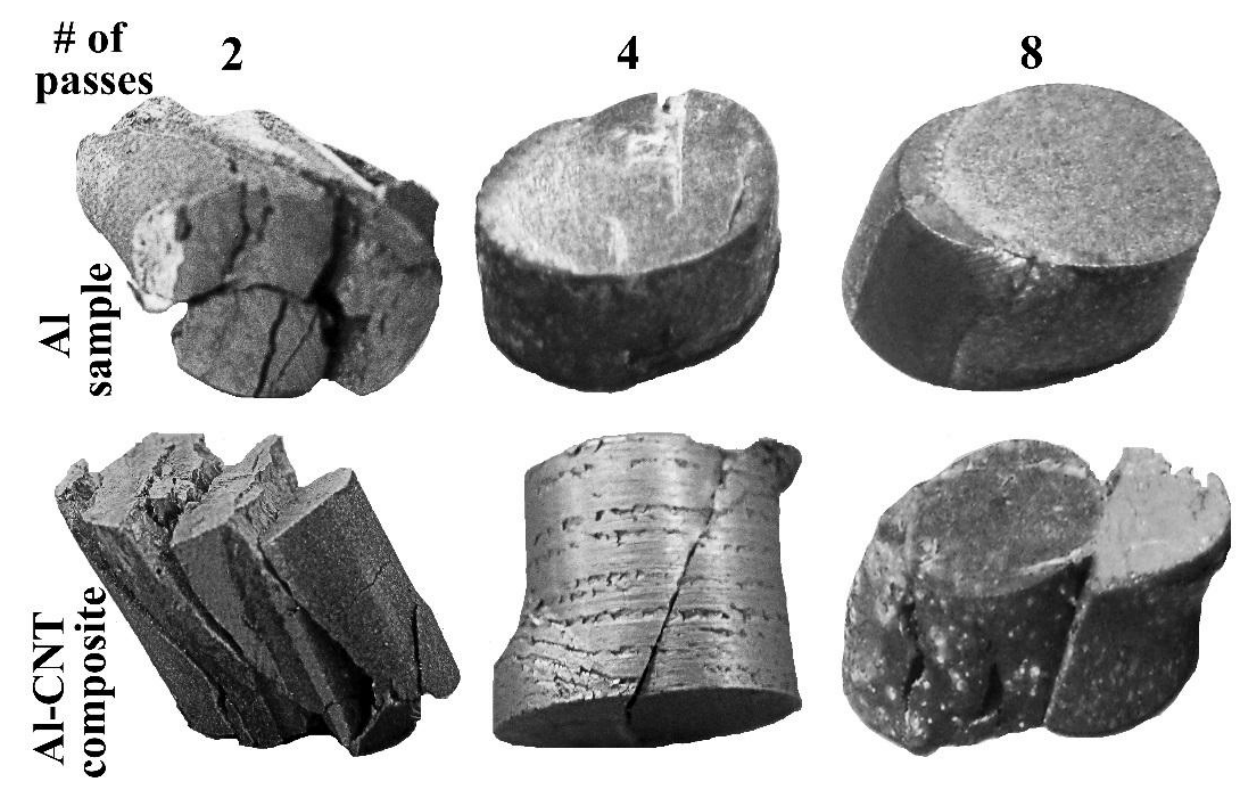

Figure 5 Compressed samples of pure Al and Al-CNT composite after a different number of ECAP passes.

\subsection{Shear punch tests}

The results of shear punch tests (SPTs) on the samples of Al and Al-CNT composite after ECAP process are presented in Fig. 6. There is no standard procedure for determining the yield point in an SPT. However, three procedures have been proposed by different investigators: (1) initial deviation from linearity along the elastic loading line, which could be viewed as $\approx 0 \%$ strain offset; (2) $1 \%$ offset in the shear strain $\gamma$, which is defined using the pure shear approximation; (3) $1 \%$ offset in the normalized displacement $\delta / t$, where $\delta$ is the punch 
displacement. It can be seen that similar to a conventional tensile stress-strain curve, after a linear elastic behavior, the curves deviates from linearity before reaching a maximum point. At this point, strain hardening associated with the thinning of the slit of the specimen between the punch and die occur simultaneously. At the peak load, the rate of thinning overtakes the rate of strain hardening, and failure initiates. Using that yield offset at $\delta=1 \%$ leads to shear yield strength value close to $1 / \sqrt{3}$ of tensile yield strength, as predicted from the von Mises criterion $[61,62]$. Following Guduru et al, 1\% yield offset has been considered for determining shear yield strength values (SYS) for all specimens; SYS has been estimated here by extrapolating the elastic line to the abscissa and taking an offset of $1 \%$ parallel to the former line (dotted black lines in Fig. 6). The maxima values in the $\tau$ vs. $\delta$ plots for the different types of specimens are referred to as the ultimate shear strength (USS). The average values of the SYS and USS of the samples at a different number of passes are summarized in Table 2. As can be seen, the USS of $\mathrm{Al}$ and Al-CNT composite samples with the ECAP passes increased significantly. For example, in the case of $\mathrm{Al}$, the USS value started at $79 \mathrm{MPa}$ and reached to $117 \mathrm{MPa}$ after 2 and 8 passes, while the composite samples were between 92 and $132 \mathrm{MPa}$ for 2 and 8 passes. The increase in both the SYS and USS value occur with increasing the number of passes, which is explained by the finer crystalline structure and dislocation density in the composite samples rather than the Al ones. Based on the Hall-Petch theory, the finer grain size results in a higher strength [30]. In summary, the better mechanical behavior of Al composite samples over the pure Al samples is explained by the increased level of grain boundaries due to finer grain structure (see Fig. 2a), a high density of dislocations (see Fig. 2c). Additionally, the uniform distribution CNTs leads to good adhesion on Al matrix after the ECAP process.

In Table 2, the $0.2 \%$ offset compressive yield stresses are also tabulated. Despite earlier observations for several other metals [4, 42], a linear correlation between the shear yield stress and yield stress was not found for the material processed here using 2 and 4 ECAP passes. This was justified by the presence of porosities in the structure. The 8 ECAP pass samples approximately obeyed $1 / \sqrt{3}$ von Mises relationship between the shear and normal strength [61, 62], which shows that after elimination of the porosities to a larger extent, the consolidated material behave like the bulk dense material. 


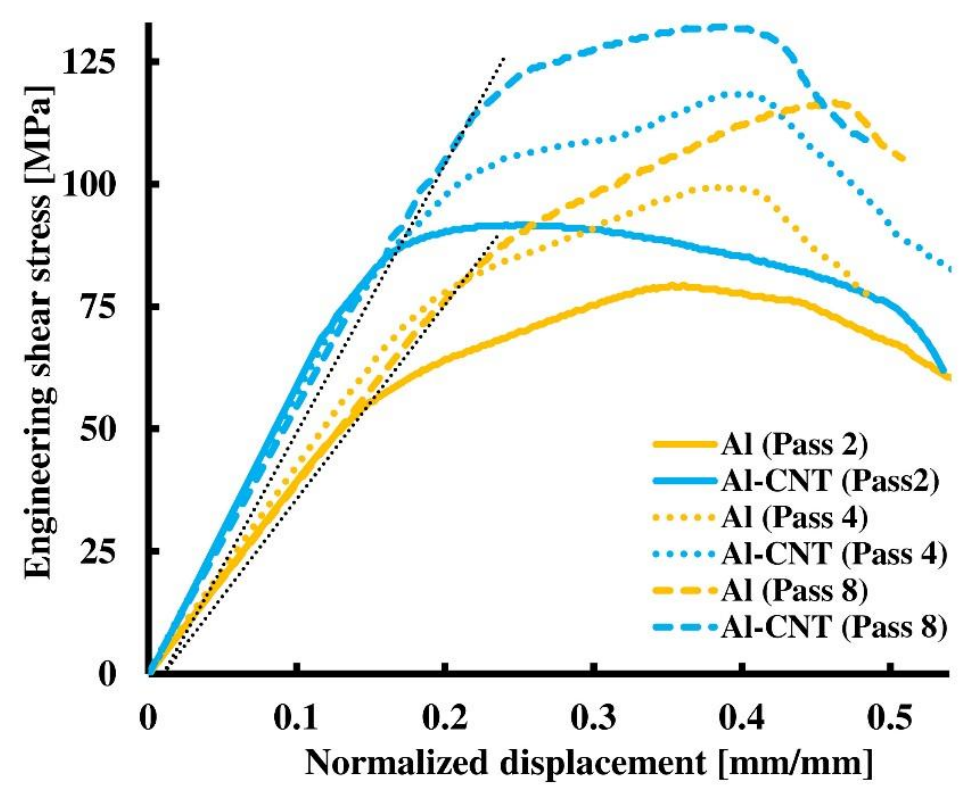

Figure 6 Shear stress-normalized displacement curves of shear punch test samples produced by ECAP. The black dotted lines show the $1 \%$ offset.

Table 2 Mechanical properties of $\mathrm{Al}$ and Al-CNT composite samples.

\begin{tabular}{|c|c|c|c|c|c|c|}
\hline $\begin{array}{c}\text { Mechanical } \\
\text { properties }\end{array}$ & \multicolumn{2}{|c|}{$\begin{array}{c}\text { Shear yield } \\
\text { strength (MPa) }\end{array}$} & \multicolumn{2}{c|}{$\begin{array}{c}\text { Ultimate shear } \\
\text { strength (MPa) }\end{array}$} & \multicolumn{2}{c|}{$\begin{array}{c}\text { Compressive yield } \\
\text { stress (MPa) }\end{array}$} \\
\hline \# of passes & Al & Al-CNT & Al & Al-CNT & Al & Al-CNT \\
\hline 2 & 57 & 85 & 79 & 92 & 42 & 47 \\
\hline 4 & 75 & 87 & 99 & 119 & 70 & 125 \\
\hline 8 & 81 & 94 & 117 & 132 & 97 & 156 \\
\hline
\end{tabular}

\subsection{Fractography after SPT}

It is generally accepted that the mechanical properties of the composites are dominated not only by the reinforcement and the matrix, but also by the interfacial bonding strength between them. It is necessary to study the material's fracture behavior to understand the load transfer between the matrix and reinforcement. Improvements to the strength of Al-CNT matrix composites were also governed by the load transfer from the matrix through the interface. To 
ensure a good load transfer, the composite must maintain a strong interfacial bonding [25, 27, $63]$.

In Figs. 7 and 8, the fracture surfaces of $\mathrm{Al}$ and Al-CNT composite samples are shown, respectively. In the materials processed using 2 ECAP passes, the powders were not consolidated well and damage quickly formed and propagated. The shallow stretched dimples caused by the severe shearing are observed. In the material after 4 ECAP passes, brittle fracture cleavage river patterns are more evident. The brittle fracture predominates especially in the samples processed by 2 and 4 ECAP passes. The failure mode was considered to be the more ductile one as the number of passes increases to 8 .
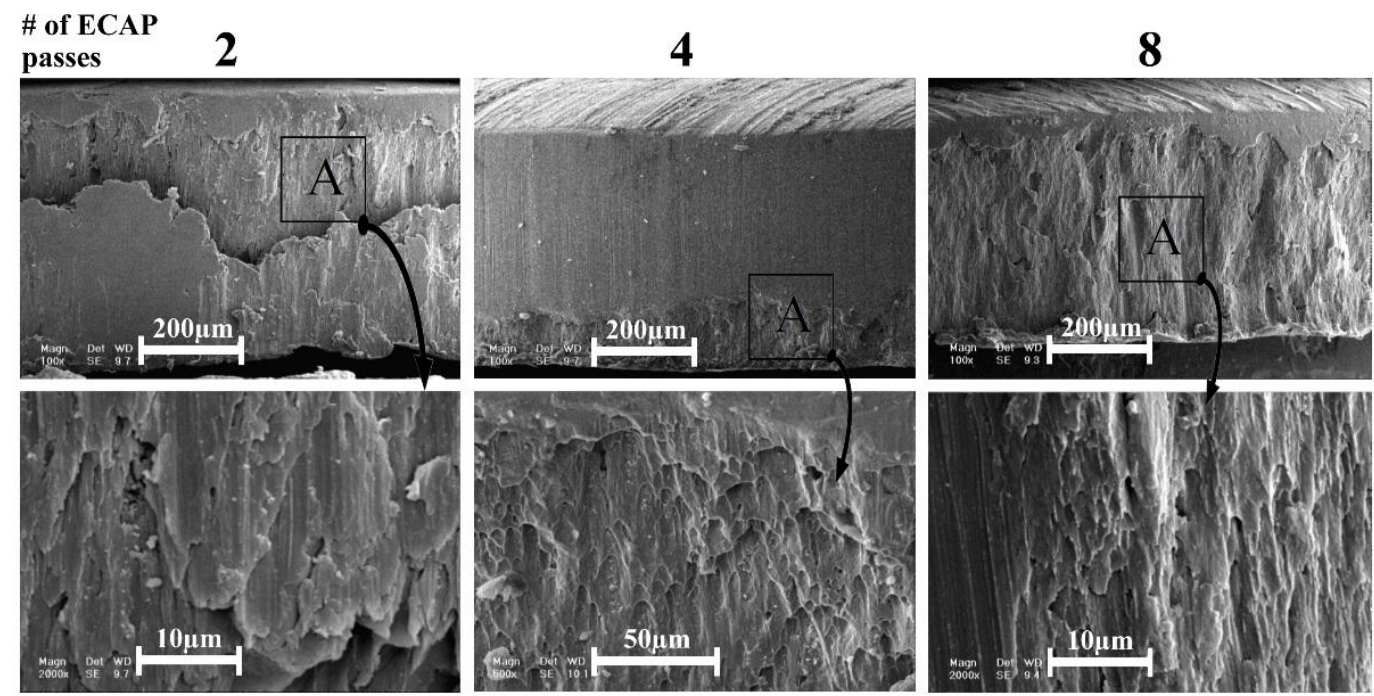

Figure 7 SEM micrographs of fracture surfaces of Al samples after SPT at different passes. The top row shows the whole fractured area and the bottom row shows the magnified selected area A.

The ductile fracture is largely controlled by void nucleation, growth, and coalescence in the matrix. In the presence of shear stress, void growth does not play an important role in the failure. In contrast, the accumulation of shear localization is leading to the formation of small stretched dimples followed by fracture. The material initially work hardens producing a localized structure of high dislocation density [23, 24, 26, 63]. 
In Fig. 8, the failures of the composite samples exhibit the brittle characteristics. In fact, because of the CNTs in the matrix, failures occurred to be brittle. In summary, the composite samples exhibit more strength and less ductility than the Al samples.

Unlike the brittle 8 pass Al-CNT samples, the dimples were observed in the Al 8 pass sample, and the nature of failure of the inter-connecting ligament had some characteristics of ductile tearing. The fine dimples occurred from the region of the matrix indicating that the $\mathrm{Al}$ matrix had undergone some ductile tearing.
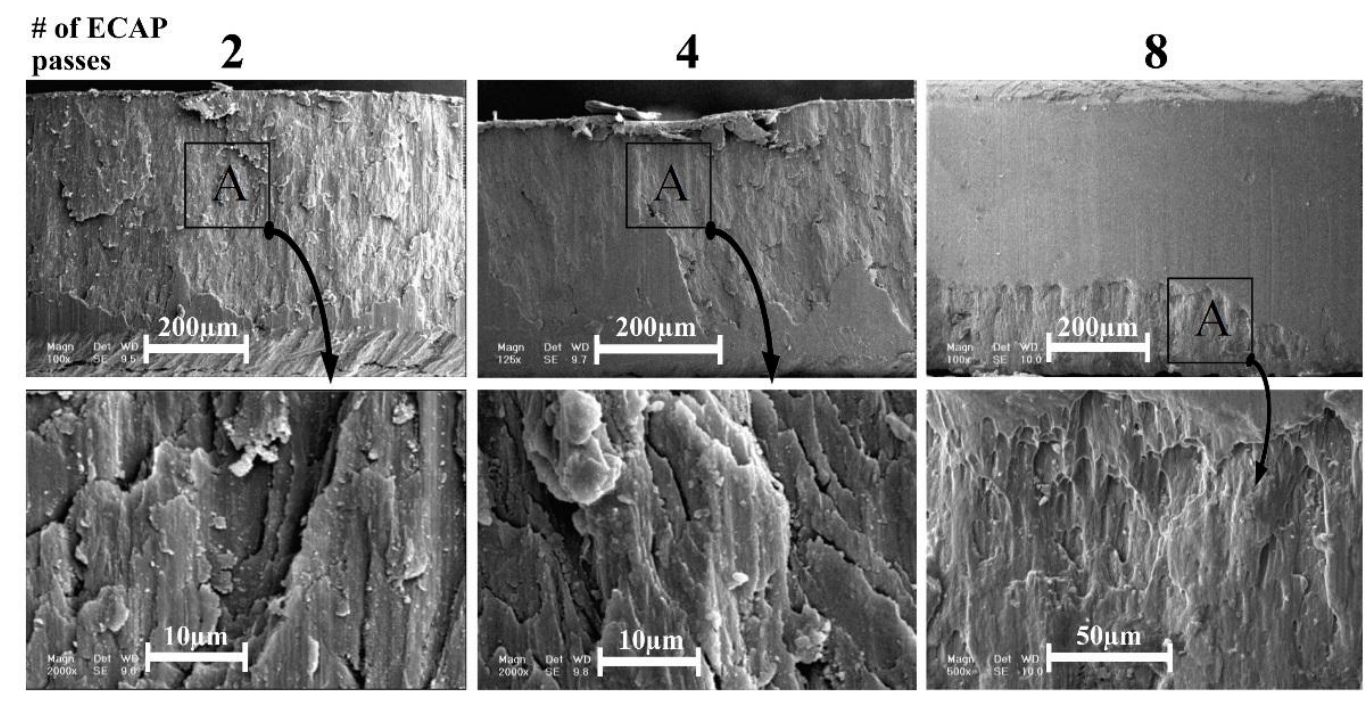

Figure 8 SEM micrographs of fracture surfaces of Al-CNT samples after SPT at different passes. The top row shows the whole fractured area and the bottom row shows the magnified selected area A.

The mode of fracture, however, depends on the cohesion force between the reinforcement particles and the matrix. The above observations and the general appearance of the fracture profiles indicate that the matrix failure plays a dominant role in the failure mechanism of this material. The reinforcement particles contribute to the failure process primarily by imposing high levels of constraint on matrix deformation and by raising the stress in the matrix to a considerable extent, much higher than that normally associated with matrix failure. Also, the shape of the CNT particles may contribute to severe stress concentration in the matrix. In view of 
the dominant role played by the matrix in the failure of the composite, it can be concluded that the selection of the matrix alloy is of paramount importance.

Interestingly, CNTs appear pulled out from the fracture surfaces of composite specimens as depicted in Fig. 9. The pits on the fracture surface (Fig. 8 after 8 pass) indicate that the length of the CNTs pulled out are very short, suggesting a strong interfacial bonding between carbon nanotubes and the Al matrix [64]. This also indicates that the high mechanical properties of AlCNT composite result from the need for interfacial deboning accompanied by the frictional pulling-out process. It is believed that this process will influence the material toughness, which will be considered in future work.
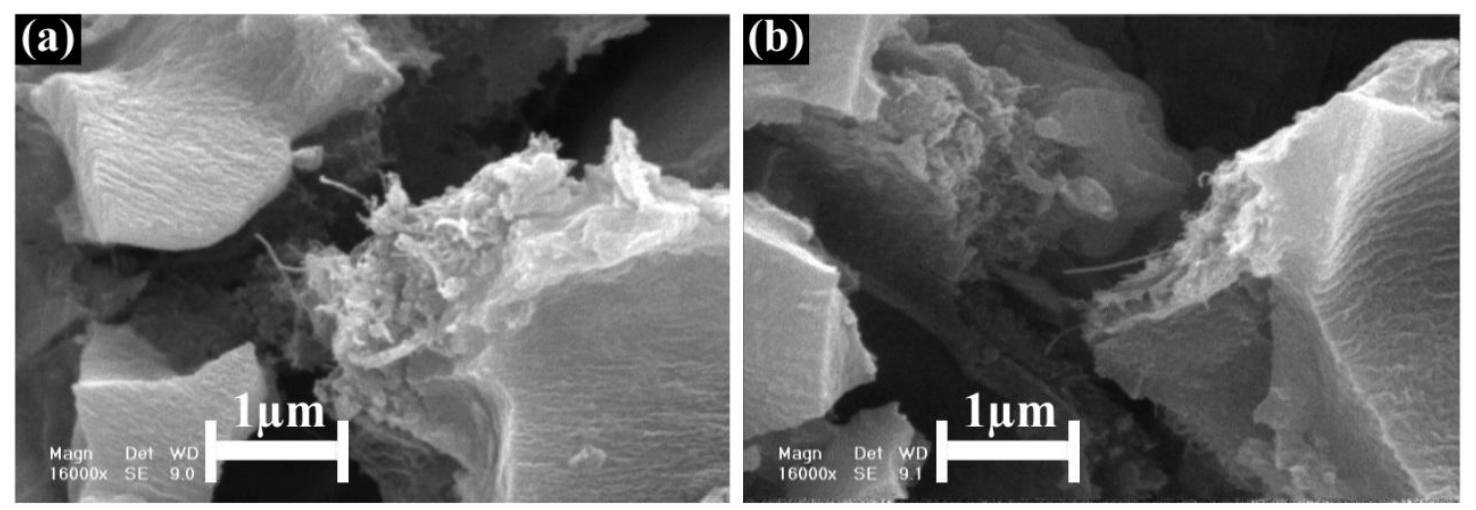

Figure 9 SEM micrographs of the composites fracture surfaces showing CNTs pull out from the adjacent particles.

\section{Conclusion}

CNT reinforced Al composites are successfully synthesized using ECAP. It was found that 8 ECAP passes are necessary to achieve the density of the composite where the effect of CNTs in enhancing the mechanical properties become significant. After 8 ECAP passes, the material exhibited the characteristics of a bulk material in terms of strain hardening and underlining mechanisms of plasticity and fracture. Mechanical behavior examined in compression and shear shows that the sufficiently densified composites, with only 2 vol. $\%$ of CNTs, can enhance these properties in pure Al for approximately 30\%. The Al-CNT composite material was found to have a smaller crystallite size and a higher content of stored dislocation density than pure Al in the same condition. Therefore, it is possible to explain the observed 
improvements in strength. The fractographic analysis revealed that the fracture surfaces of the composite exhibit characteristics of more brittle behavior than pure Al.

\section{Acknowledgements}

HZ, MRT, and MM would like to acknowledge the financial support of Isfahan University of Technology. MK gratefully acknowledges the support by the US National Science Foundation under grant No. CMMI-1541918.

\section{References}

[1] T.H. Nam, G. Requena, P. Degischer, Thermal expansion behaviour of aluminum matrix composites with densely packed $\mathrm{SiC}$ particles, Composites Part A: Applied Science and Manufacturing 39(5) (2008) 856-865.

[2] M. Jahedi, B. Mani, S. Shakoorian, E. Pourkhorshid, M. Hossein Paydar, Deformation rate effect on the microstructure and mechanical properties of Al-SiCp composites consolidated by hot extrusion, Materials Science and Engineering: A 556 (2012) 23-30.

[3] M. Jahedi, M.H. Paydar, M. Knezevic, Enhanced microstructural homogeneity in metalmatrix composites developed under high-pressure-double-torsion, Materials Characterization 104 (2015) 92-100.

[4] M. Zabihi, M.R. Toroghinejad, A. Shafyei, Evaluating the mechanical behavior of hot rolled Al/alumina composite strips using shear punch test, Materials Science and Engineering: A 618 (2014) 490-495.

[5] Y. Yao, L. Chen, Processing of B4C Particulate-reinforced Magnesium-matrix Composites by Metal-assisted Melt Infiltration Technique, Journal of Materials Science \& Technology 30(7) (2014) 661-665.

[6] M.R. Akbarpour, M. Farvizi, D.J. Lee, H. Rezaei, H.S. Kim, Effect of high-pressure torsion on the microstructure and strengthening mechanisms of hot-consolidated $\mathrm{Cu}-\mathrm{CNT}$ nanocomposite, Materials Science and Engineering: A 638 (2015) 289-295.

[7] M.R. Basariya, V. Srivastava, N. Mukhopadhyay, Microstructural characteristics and mechanical properties of carbon nanotube reinforced aluminum alloy composites produced by ball milling, Materials \& Design 64 (2014) 542-549. 
[8] Q. Liu, L. Ke, F. Liu, C. Huang, L. Xing, Microstructure and mechanical property of multiwalled carbon nanotubes reinforced aluminum matrix composites fabricated by friction stir processing, Materials \& Design 45 (2013) 343-348.

[9] K. Fukuchi, K. Sasaki, K. Katagiri, T. Imanishi, A. Kakitsuji, Aluminium based high thermal conductive composites containing CNT and VGCF-deformation dependence of thermal conductivity, Procedia Engineering 10 (2011) 912-917.

[10] M. Tan, X. Zhang, Powder metal matrix composites: selection and processing, Materials Science and Engineering: A 244(1) (1998) 80-85.

[11] T. Clyne, P. Withers, An introduction to metal matrix composites, Cambridge University Press 1995.

[12] I. Sabirov, O. Kolednik, R. Valiev, R. Pippan, Equal channel angular pressing of metal matrix composites: effect on particle distribution and fracture toughness, Acta materialia 53(18) (2005) 4919-4930.

[13] P. Quang, Y.G. Jeong, S.C. Yoon, S.H. Hong, H.S. Kim, Consolidation of 1 vol.\% carbon nanotube reinforced metal matrix nanocomposites via equal channel angular pressing, Journal of Materials Processing Technology 187-188 (2007) 318-320.

[14] M. Ardeljan, M. Knezevic, T. Nizolek, I. Beyerlein, S. Zheng, J. Carpenter, R. McCabe, N. Mara, T. Pollock, A multi-scale model for texture development in $\mathrm{Zr} / \mathrm{Nb}$ nanolayered composites processed by accumulative roll bonding, IOP Conference Series: Materials Science and Engineering, IOP Publishing, 2014, p. 012170.

[15] M. Knezevic, T. Nizolek, M. Ardeljan, I.J. Beyerlein, N.A. Mara, T.M. Pollock, Texture evolution in two-phase $\mathrm{Zr} / \mathrm{Nb}$ lamellar composites during accumulative roll bonding, International Journal of Plasticity 57(0) (2014) 16-28.

[16] M. Knezevic, I.J. Beyerlein, T. Nizolek, N.A. Mara, T.M. Pollock, Anomalous Basal Slip Activity in Zirconium under High-strain Deformation, Materials Research Letters 1(3) (2013) 133-140.

[17] M. Ardeljan, M. Knezevic, T. Nizolek, I.J. Beyerlein, N.A. Mara, T.M. Pollock, A study of microstructure-driven strain localizations in two-phase polycrystalline $\mathrm{HCP} / \mathrm{BCC}$ composites using a multi-scale model, International Journal of Plasticity 74 (2015) 35-57.

[18] Y. Xue, B. Jiang, L. Bourgeois, P. Dai, M. Mitome, C. Zhang, M. Yamaguchi, A. Matveev, C. Tang, Y. Bando, Aluminum matrix composites reinforced with multi-walled boron nitride 
nanotubes fabricated by a high-pressure torsion technique, Materials \& Design 88 (2015) 451460.

[19] M. Jahedi, M.H. Paydar, Three-dimensional finite element analysis of torsion extrusion (TE) as an SPD process, Materials Science and Engineering: A 528(29) (2011) 8742-8749.

[20] R.Y. Lapovok, The role of back-pressure in equal channel angular extrusion, Journal of Materials Science 40(2) (2005) 341-346.

[21] B. Mani, M. Jahedi, M.H. Paydar, Consolidation of commercial pure aluminum powder by torsional-equal channel angular pressing (T-ECAP) at room temperature, Powder Technology 219 (2012) 1-8.

[22] M. Jahedi, M.H. Paydar, S. Zheng, I.J. Beyerlein, M. Knezevic, Texture evolution and enhanced grain refinement under high-pressure-double-torsion, Materials Science and Engineering: A 611 (2014) 29-36.

[23] M. Rezayat, A. Akbarzadeh, A. Owhadi, Production of high strength Al-Al 2 O 3 composite by accumulative roll bonding, Composites Part A: Applied Science and Manufacturing 43(2) (2012) 261-267.

[24] J. Onoro, M. Salvador, L. Cambronero, High-temperature mechanical properties of aluminium alloys reinforced with boron carbide particles, Materials Science and Engineering: A 499(1) (2009) 421-426.

[25] S. Sharma, B. Girish, D. Somashekar, R. Kamath, B. Satish, Mechanical properties and fractography of zircon-particle-reinforced ZA-27 alloy composite materials, Composites science and technology 59(12) (1999) 1805-1812.

[26] I. Stone, P. Tsakiropoulos, The effect of reinforcement on the notched and unnotched room temperature tensile properties of $\mathrm{Al}-4 \mathrm{wt} . \% \mathrm{Cu} / \mathrm{SiC} \mathrm{p} \mathrm{MMCs}$, Materials Science and Engineering: A 241(1) (1998) 19-29.

[27] B.-K. Hwu, S.-J. Lin, M.-T. Jahn, The interfacial compounds and SEM fractography of squeeze-cast SiC p/6061 Al composites, Materials Science and Engineering: A 206(1) (1996) 110-119.

[28] Y. Iwahashi, Z. Horita, M. Nemoto, T.G. Langdon, An investigation of microstructural evolution during equal-channel angular pressing, Acta Materialia 45(11) (1997) 4733-4741.

[29] Y. Iwahashi, Z. Horita, M. Nemoto, T.G. Langdon, The process of grain refinement in equal-channel angular pressing, Acta Materialia 46(9) (1998) 3317-3331. 
[30] V.M. Segal, Materials processing by simple shear, Materials Science and Engineering: A 197(2) (1995) 157-164.

[31] G. Williamson, W. Hall, X-ray line broadening from filed aluminium and wolfram, Acta metallurgica 1(1) (1953) 22-31.

[32] R. Jamaati, M.R. Toroghinejad, J. Dutkiewicz, J.A. Szpunar, Investigation of nanostructured Al/Al2O3 composite produced by accumulative roll bonding process, Materials \& Design 35 (2012) 37-42.

[33] J. Ghosh, S.K. Chattopadhayay, A.K. Meikap, S.K. Chatterjee, Microstructure characterization of titanium-base aluminium alloys by X-ray diffraction using Warren-Averbach and Rietveld method, Journal of Alloys and Compounds 453(1-2) (2008) 131-137.

[34] L. Lutterotti, P. Scardi, Simultaneous structure and size-strain refinement by the Rietveld method, Journal of applied Crystallography 23(4) (1990) 246-252.

[35] A. Chanda, H. Pal, M. De, S. Kajiwara, T. Kikuchi, Characterisation of microstructure of isothermal martensite formed at low temperature in powder of $\mathrm{Fe}-23 \mathrm{Ni}-3.3 \mathrm{Mn}$ alloy by Rietveld method, Materials Science and Engineering: A 265(1) (1999) 110-116.

[36] H. Pal, A. Chanda, M. De, Characterisation of microstructure of isothermal martensite in Fe-23Ni-3.8 Mn by Rietveld method, Journal of alloys and compounds 278(1) (1998) 209-215.

[37] B.H. Toby, R factors in Rietveld analysis: how good is good enough?, Powder diffraction 21(01) (2006) 67-70.

[38] M. Zecevic, M. Knezevic, A dislocation density based elasto-plastic self-consistent model for the prediction of cyclic deformation: Application to AA6022-T4, International Journal of Plasticity 72 (2015) 200-217.

[39] D.H. Smith, J. Bicknell, L. Jorgensen, B.M. Patterson, N.L. Cordes, I. Tsukrov, M. Knezevic, Microstructure and mechanical behavior of direct metal laser sintered Inconel alloy 718, Materials Characterization 113 (2016) 1-9.

[40] M. Knezevic, A. Levinson, R. Harris, R.K. Mishra, R.D. Doherty, S.R. Kalidindi, Deformation twinning in AZ31: influence on strain hardening and texture evolution, Acta Materialia 58(19) (2010) 6230-6242.

[41] H.R. Abedi, A. Zarei-Hanzaki, M. Bagherzadeh Biucki, M. Emamy, Evaluating the room temperature mechanical properties of age hardened AZ80 magnesium alloy using shear punch testing method, Materials Science and Engineering: A 606 (2014) 360-369. 
[42] R.K. Guduru, K.A. Darling, R. Kishore, R.O. Scattergood, C.C. Koch, K.L. Murty, Evaluation of mechanical properties using shear-punch testing, Materials Science and Engineering: A 395(1-2) (2005) 307-314.

[43] R.K. Guduru, A.V. Nagasekhar, R.O. Scattergood, C.C. Koch, K.L. Murty, Thickness and clearance effects in shear punch testing, Advanced Engineering Materials 9(3) (2007) 157.

[44] V. Stolyarov, R. Lapovok, I. Brodova, P. Thomson, Ultrafine-grained Al-5 wt.\% Fe alloy processed by ECAP with backpressure, Materials Science and Engineering: A 357(1) (2003) 159-167.

[45] D. Tabor, A simple theory of static and dynamic hardness, Proceedings of the Royal Society of London A: Mathematical, Physical and Engineering Sciences, The Royal Society, 1948, pp. 247-274.

[46] B.D. Cullity, J.W. Weymouth, Elements of X-ray Diffraction, American Journal of Physics 25(6) (1957) 394-395.

[47] J.B. Cohen, J.E. Hilliard, Local atomic arrangements studied by X-ray diffraction, (1967).

[48] H.P. Klug, L.E. Alexander, X-ray diffraction procedures, Wiley New York1954.

[49] P. Thompson, D. Cox, J. Hastings, Rietveld refinement of Debye-Scherrer synchrotron Xray data from Al2O3, Journal of Applied Crystallography 20(2) (1987) 79-83.

[50] A.P. Zhilyaev, D.L. Swisher, K. Oh-ishi, T.G. Langdon, T.R. McNelley, Microtexture and microstructure evolution during processing of pure aluminum by repetitive ECAP, Materials Science and Engineering: A 429(1-2) (2006) 137-148.

[51] B.Q. Han, T.G. Langdon, Achieving enhanced tensile ductility in an Al-6061 composite processed by severe plastic deformation, Materials Science and Engineering: A 410-411 (2005) 430-434.

[52] I. Sabirov, Y. Estrin, M.R. Barnett, I. Timokhina, P.D. Hodgson, Tensile deformation of an ultrafine-grained aluminium alloy: Micro shear banding and grain boundary sliding, Acta Materialia 56(10) (2008) 2223-2230.

[53] J.A. Hines, K.S. Vecchio, S. Ahzi, A model for microstructure evolution in adiabatic shear bands, Metallurgical and Materials Transactions A 29(1) (1998) 191-203.

[54] N. Hansen, X. Huang, R. Ueji, N. Tsuji, Structure and strength after large strain deformation, Materials Science and Engineering: A 387-389 (2004) 191-194. 
[55] M. Reihanian, R. Ebrahimi, N. Tsuji, M.M. Moshksar, Analysis of the mechanical properties and deformation behavior of nanostructured commercially pure Al processed by equal channel angular pressing (ECAP), Materials Science and Engineering: A 473(1-2) (2008) 189194.

[56] E.A. El-Danaf, M.S. Soliman, A.A. Almajid, M.M. El-Rayes, Enhancement of mechanical properties and grain size refinement of commercial purity aluminum 1050 processed by ECAP, Materials Science and Engineering: A 458(1-2) (2007) 226-234.

[57] G. Ramu, R. Bauri, Effect of equal channel angular pressing (ECAP) on microstructure and properties of Al-SiCp composites, Materials \& Design 30(9) (2009) 3554-3559.

[58] M.S. Arab, N. El Mahallawy, F. Shehata, M.A. Agwa, Refining SiCp in reinforced Al-SiC composites using equal-channel angular pressing, Materials \& Design 64 (2014) 280-286.

[59] X. Long, Y. Bai, M. Algarni, Y. Choi, Q. Chen, Study on the strengthening mechanisms of Cu/CNT nano-composites, Materials Science and Engineering: A 645 (2015) 347-356.

[60] J.G. Park, D.H. Keum, Y.H. Lee, Strengthening mechanisms in carbon nanotube-reinforced aluminum composites, Carbon 95 (2015) 690-698.

[61] G.E. Dieter, D. Bacon, Mechanical Metallurgy, McGraw-Hill1988.

[62] W.F. Hosford, R.M. Caddell, Metal forming: mechanics and metallurgy, Cambridge University Press2011.

[63] Y. Chen, B. Liu, X. He, Y. Huang, K. Hwang, Failure analysis and the optimal toughness design of carbon nanotube-reinforced composites, Composites Science and Technology 70(9) (2010) 1360-1367.

[64] Q. Qin, J. Ye, Toughening mechanisms in composite materials, Elsevier2015. 


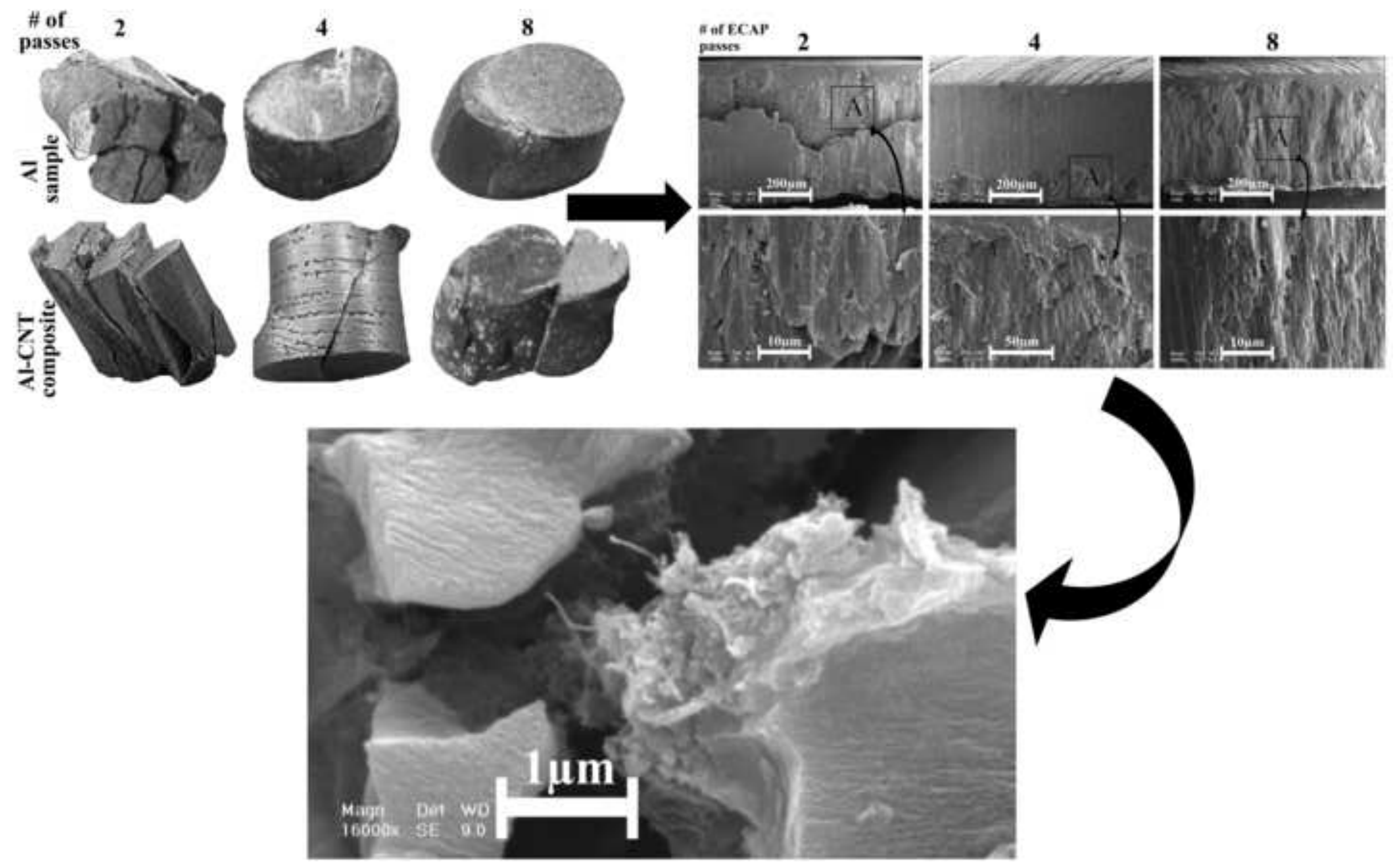

